\title{
Existe convergência espacial da produtividade agrícola no Brasil?
}

\author{
Eduardo Simões de Almeida* \\ Fernando Salgueiro Perobelli** \\ Pedro Guilherme Costa Ferreira***
}

RESUMO: Este trabalho analisa a convergência espacial da produtividade agrícola da terra para as microrregiões brasileiras entre o período de 1991 a 2003. Tal análise avalia se há evidências para a existência de convergência $\beta$ da produtividade agrícola, controlando-se explicitamente para efeitos espaciais. Para tanto, conduziu-se preliminarmente uma análise exploratória de dados espaciais, que detectou a presença de autocorrelação espacial para a produtividade da terra. Numa etapa posterior, desenvolveramse modelos econométricos espaciais para a análise da convergência.

Palavras-chave: $\beta$ convergência; produtividade da terra; econometria espacial; autocorrelação espacial.

Classificação JEL: R12, C31, Q10.

Abstract: This work analyzes the convergence of land productivity for Brazilian regions from 1991 to 2003. It evaluates if there is or not $\beta$ convergence of the agricultural productivity, controlling explicitly for spatial

\footnotetext{
* Professor Adjunto da FEA/UFJF e pesquisador do CNPq email: eduardo.almeida@ufjf.edu.br

** Professor Adjunto da FEA/UFJF e pesquisador do CNPq email: fernando.perobelli@ufjf.edu.br

*** Mestrando em Economia do Curso de Mestrado em Economia Aplicada FEA/UFJF email: pgcferreira@gmail.com
} 
effects. To do so, an exploratory spatial data analysis was previously implemented and the presence of spatial autocorrelation for the land productivity was detected. In addition, spatial econometric models were developed for the convergence analysis.

Keywords: $\beta$ convergence; land productivity, spatial econometrics, spatial autocorrelation.

JEL Classification: R12, C31, Q10.

\section{Introdução}

A importância da agricultura para o crescimento econômico tende a ser desigual para as diferentes regiões. Segundo Johnston \& Mellor (1961), as funções da agricultura podem ser listadas como: a) fornecer alimentos; b) prover capital, especialmente para a expansão do setor não-agrícola; c) oferecer mão-de-obra para o crescimento e diversificação de atividades na economia; d) gerar ganhos cambiais; e, e) constituir mercado para os produtos do setor não-agrícola. Na visão de Johnston \& Mellor (1961), as funções desempenhadas pela agricultura estão relacionadas aos primeiros estágios de desenvolvimento econômico. Isso ocorre, em parte, devido à diminuição da participação da agricultura no produto e no emprego regional. No entanto, a agricultura pode contribuir para o desenvolvimento da economia como um todo, mesmo nos estágios mais avançados.

Deste modo, fica evidente que analisar o comportamento da agricultura é de suma importância para a economia de um País, principalmente no Brasil, onde o setor participa de forma significativa tanto nas exportações quanto no mercado de trabalho. Uma das maneiras de avaliar tal desempenho é acompanhar o comportamento da produtividade agrícola ao longo do tempo e através das regiões, avaliando se a sua evolução mostra convergência. Conforme Lopes (2004), "a convergência é um processo em que uma mesma variável (por exemplo, renda per capita ou produtividade da terra) apresenta diferentes valores entre países, regiões ou estados, mas essa diferença se reduz ao longo do tempo, indicando que a desigualdade diminui”. 
A discussão de convergência da renda atraiu muitos estudiosos e existe um grande número de trabalhos empíricos que discutem essa questão. Em outros termos, a questão do crescimento mais rápido das regiões mais pobres do que as mais ricas foi exaustivamente discutida na literatura. ${ }^{1}$ O trabalho seminal de Baumol (1986) examinou a convergência no período de 1870 a 1979 entre 16 países industrializados, conforme a seguinte expressão:

$$
\ln (Y / N)_{i, t}-\ln (Y / N)_{i, t-1}=\alpha+\beta \ln (Y / N)_{i, t-1}+\varepsilon_{i}
$$

em que $\ln (Y / N)$ é o logaritmo da renda per capita, $\varepsilon$ é o termo de erro e $i$ é o indexador para os diversos países.

Segundo Baumol (1986), se existir convergência absoluta, $\beta$ será negativo, ou seja, os países com renda inicial maior terão menores taxas de crescimento. Com isso, Baumol procura mostrar que ao longo de um período de tempo, as rendas dos diversos países estariam convergindo para uma renda comum. Portanto, se os países de rendas menores crescem mais, a tendência é que, ceteris paribus, tais rendas se igualem no tempo. Cabe ressaltar que muito se avançou na discussão sobre a convergência absoluta desde o trabalho de Baumol (1986). Todavia, não é do escopo deste artigo discutir todas as variantes desta linha de pesquisa. ${ }^{2}$

É importante elencar a existência de trabalhos, como o de Rey e Montouri (1999), que aplicam a análise da convergência da renda, adotando a abordagem da econometria espacial. Dall'erba (2003) estima a convergência da produtividade do trabalho com base em 48 regiões da Espanha, durante os anos de 1980-1996. Entre os artigos relacionados ao tema, no Brasil, destaca-se a tese de Lopes (2004), que analisa a evolução da produtividade da terra das onze principais culturas brasileiras, avaliando o processo de convergência dessa variável entre os estados produtores.

${ }^{1}$ Embora a maioria dos trabalhos que analisou a questão da convergência de renda encontrou evidências de convergência com nivelamento para cima, é possível, teórica e empiricamente, admitir a existência de convergência com nivelamento para baixo. Todavia, como ficará claro adiante, não é razoável admitir convergência com nivelamento para baixo da produtividade agrícola no Brasil no período analisado.

${ }^{2}$ Consultar Barro e Sala-i-Martin (1995) para uma discussão avançada sobre as convergências absoluta e condicional. 
Magalhães et al. (2000), por sua vez, discute a convergência da renda através dos estados brasileiros durante o período de 1970 a 1995.

A investigação de produtividade média agrícola sob o prisma do modelo de $\beta$ convergência, controlando-se para os efeitos espaciais, é explorada no presente trabalho de forma inédita tanto para o Brasil quanto para outros países. Assim, o objetivo deste trabalho é adaptar a idéia apresentada por Baumol (1986) para entender o comportamento da produtividade média agrícola na terra. Nesse sentido, ao formalizar uma equação de convergência absoluta a la Baumol para o índice de produtividade média agrícola na terra, buscar-se-á verificar se as regiões com menor grau de produtividade agrícola estão convergindo para as regiões com maior grau de produtividade. Enfim, a idéia de utilizar a análise de convergência no trabalho é testar se os índices de produtividade agrícola estão ficando mais homogêneos no país.

De acordo com Lopes (2004), existem basicamente três razões para se verificar a convergência da produtividade agrícola, a saber, a ocorrência de mudanças estruturais no processo de produção (vinculadas, sobretudo a inovações tecnológicas e/ou incentivadas por políticas públicas, como, por exemplo, o processo de apreciação do câmbio na maior parte dos anos noventa e a abertura econômica, entre outros); o fenômeno da difusão tecnológica de novas sementes, novos sistemas de plantio, novos implementos agrícolas etc; e a eliminação de obstáculos ao crescimento da produção, como restrições ambientais, falta de adequada logística e carência de financiamento, dentre outros.

Outro aspecto relevante no artigo é o período selecionado para a análise (1991 a 2003). Esse período pode ser considerado promissor para a agricultura em termos de expansão da produção e de obtenção de ganhos de produtividade. Sem sombra de dúvida, neste período, consolida-se a posição do País como um dos mais importantes e competitivos produtores agrícolas do mundo.

Ademais, esse intervalo de tempo testemunhou várias mudanças estruturais que marcaram indelevelmente o desenvolvimento agrícola no Brasil, tais como a abertura econômica, a estabilização dos preços, a apreciação cambial (1994-1999) e a posterior depreciação cambial (a partir de fevereiro de 1999) que acarretou a expansão extraordinária das exportações, impulsionada também pela expansão do crescimento 
econômico do mundo. Deste modo, torna-se relevante dividir a estimativa da convergência em sub-períodos que abrangem tais medidas macroeconômicas.

A primeira estimativa testa se no período de 1991 a 1994, existe convergência da produtividade média agrícola. Isso porque esse é o período do início da abertura econômica brasileira até a adoção do Plano Real. Nessa época, de acordo com documento do DIEESE (2004), a moeda interna estava desvalorizada em relação ao dólar, constituindo um processo de superinflação crônica.

A segunda estimativa de convergência engloba o período de 1995 até 1999. Esse é o período após a implantação do Plano Real. Nesse período, houve um controle mais rigoroso da inflação, ao passo que o câmbio apreciou-se, transformando-se na âncora cambial da economia. É importante observar que esse é um período de altos e baixos na economia brasileira, considerando-se que, no ano de 1995, o país vivia um momento de estabilidade com crescimento econômico, fazendo com que o nível de atividade acelerasse e o desemprego diminuísse. No entanto, tal cenário econômico foi interrompido pelas sucessivas crises internacionais ocorridas no cenário mundial, que provocaram a fuga de capitais do Brasil. Isso levou o governo a promover sucessivos aumentos na taxa de juros, trazendo repercussões negativas para a economia brasileira.

A terceira estimativa de convergência aplica-se ao período de 2000 a 2003 e objetiva testar qual foi o impacto da moeda desvalorizada na produtividade média agrícola em relação ao insumo terra. Por último, a quarta estimativa engloba todo o período de 1991 a 2003 em que ocorreram profundas mudanças macroeconômicas na economia brasileira.

Outro ponto observado é a evolução da produtividade média no Brasil e nos estados ao longo dos sub-períodos. Isto porque poderia, eventualmente, ocorrer convergência da produtividade agrícola no país, caso houvesse queda na produtividade tanto no Brasil quanto nos estados, proporcionando uma conclusão enganosa dos dados. Todavia, como se verifica na Tabela 1, isso não acontece. Segundo Considera et al. (2002), são os ganhos de produtividade a principal alavanca do processo de crescimento do setor agrícola brasileiro na década de 1990. 
Tabela 1. Variação Percentual da Produtividade Média Agrícola para cada sub-período: 1991- 94; 1995-99; 2000-03; e para o período: 1991 a 2003.

\begin{tabular}{|c|c|c|c|c|}
\hline & $1991 / 94$ & $1995 / 99$ & $2000 / 03$ & $1991 / 03$ \\
\hline Brasil & 5,00 & 8,31 & 7,81 & 29,33 \\
\hline Acre & $-14,27$ & 6,05 & $-1,55$ & $-11,01$ \\
\hline Alagoas & 14,42 & 17,99 & 8,48 & 47,63 \\
\hline Amapá & $-9,53$ & 10,47 & $-6,17$ & $-17,26$ \\
\hline Amazonas & 0,26 & 37,19 & $-29,85$ & $-14,32$ \\
\hline Bahia & 2,86 & 7,74 & 4,99 & 4,52 \\
\hline Ceará & $-19,54$ & 7,02 & $-10,16$ & $-15,03$ \\
\hline Distrito Federal & $-3,15$ & 13,26 & 0,48 & 32,34 \\
\hline Espírito Santo & 20,17 & 25,90 & $-2,38$ & 37,44 \\
\hline Goiás & 7,30 & 34,22 & 9,24 & 81,52 \\
\hline Maranhão & $-4,04$ & $-14,64$ & 5,34 & $-9,39$ \\
\hline Mato Grosso & 12,70 & 17,73 & 22,72 & 84,19 \\
\hline Mato Grosso do Sul & 13,82 & 17,44 & 86,83 & 165,22 \\
\hline Minas Gerais & $-2,55$ & 10,15 & 12,69 & 31,89 \\
\hline Pará & 0,02 & 14,08 & $-11,31$ & $-0,30$ \\
\hline Paraóba & $-24,02$ & $-13,61$ & 19,40 & $-3,67$ \\
\hline Paraná & 35,52 & 13,87 & 19,65 & 87,98 \\
\hline Pernambuco & $-14,47$ & $-14,79$ & 19,14 & 1,16 \\
\hline Piauí & $-35,23$ & $-16,46$ & 0,04 & $-34,93$ \\
\hline Rio de Janeiro & $-2,52$ & 11,36 & 2,14 & 16,75 \\
\hline Rio Grande do Norte & $-16,74$ & $-10,69$ & 11,90 & 8,88 \\
\hline Rio Grande do Sul & 33,55 & $-0,32$ & 9,50 & 60,26 \\
\hline Rondônia & $-1,04$ & $-34,18$ & 15,57 & $-24,89$ \\
\hline Roraima & $-9,07$ & 9,62 & 12,51 & 18,19 \\
\hline Santa Catarina & 17,73 & 6,58 & 10,61 & 28,93 \\
\hline São Paulo & 19,64 & 10,10 & 6,91 & 50,53 \\
\hline Sergipe & $-15,44$ & 1,45 & 9,41 & $-13,71$ \\
\hline Tocantins & 7,27 & 13,68 & $-18,76$ & 5,62 \\
\hline
\end{tabular}

Fonte: Pesquisa Agrícola Municipal (PAM) do IBGE. 
Vale observar também a importância da utilização de econometria espacial nos estudos de convergência. Segundo Quah (1996), a maioria dos trabalhos sobre convergência utiliza dados regionais, enquanto grande parte dos trabalhos trata as regiões como unidades isoladas, ou seja, não considerando a interação com seu entorno. Ainda, de acordo com Rey e Montouri (1999), o desenvolvimento de ferramentas de análise exploratória de dados espaciais, bem como as técnicas econométricas espaciais, permitiu uma nova percepção da dinâmica geográfica dos padrões de crescimento da renda no tempo. ${ }^{3}$

Rey e Montouri (1999) reconheceram que um modelo de convergência, composto por dados organizados em unidades espaciais, deveria levar em consideração os efeitos espaciais que poderiam resultar da interação espacial entre os agentes.

A diferença entre a econometria espacial e a econometria convencional concentra-se na preocupação de se incorporar na modelagem o padrão da interação sócio-econômica entre os agentes num sistema (a autocorrelação espacial), assim como as características da estrutura desse sistema no espaço (a heterogeneidade espacial). Essas interações e as características estruturais geram efeitos espaciais que influenciam vários processos econômicos (Anselin, 1988; Anselin e Bera, 1998).

Não é difícil de perceber que a agricultura é muito sensível aos efeitos espaciais. O desenvolvimento das culturas agrícolas através do espaço é heterogêneo, pois depende de diferentes técnicas de produção, condições de climas, solo e topografia. Por outro lado, os efeitos de interdependência entre diferentes regiões produtoras manifestam-se de diversas formas, tais como ordenação geográfica dos dados, difusão espacial de certos fenômenos que influenciam os vizinhos, processos de competição espacial no âmbito da expansão da fronteira agrícola ou na formação de cinturões agrícolas.

Em suma, este trabalho apresenta uma análise de convergência absoluta para a produtividade média agrícola, controlando-se os efeitos espaciais. Na presença dos efeitos espaciais, os métodos econométricos

${ }^{3}$ De acordo com Anselin (1999, p. 2), "econometria espacial é um sub-campo da econometria que lida com as complicações causadas pela interação espacial (autocorrelação espacial) e pela estrutura espacial (heterogeneidade espacial), em modelos de regressão para dados na forma de seção cruzada e painel de dados". 
tradicionais levam à inferência espúria, com graves danos para a formulação de políticas públicas. Nesse sentido, busca-se verificar a existência de convergência da produtividade média para as microrregiões brasileiras, tendo dois objetivos centrais. O primeiro é fornecer uma nova percepção da dinâmica geográfica utilizando para isso métodos de análise exploratória de dados espaciais e de econometria espacial. O segundo, diz respeito à identificação e exposição do grau da convergência na produtividade agrícola que vem ocorrendo no País.

O artigo está organizado em cinco seções, incluindo esta primeira, de caráter introdutório. A próxima seção trata da descrição sucinta do banco de dados. A terceira seção traz a análise exploratória de dados espaciais. Na quarta seção, são apresentados a metodologia e os resultados das regressões de convergência, bem como sua discussão. Na última seção, são abordadas as conclusões de relevo e tecidas as considerações finais.

\section{Dados}

Este trabalho utiliza os dados da Pesquisa Agrícola Municipal do IBGE (Instituto Brasileiro de Geografia e Estatística), consultados por meio do Sistema IBGE de Recuperação Automática (SIDRA). Para a elaboração do artigo, foram compilados dados sobre a produção agrícola total e a área plantada total para os anos de 1991 a 2003 por microrregião.

Devido à incompatibilidade de unidade de medida, já que na consulta ao SIDRA não estavam disponíveis dados referentes à produção total, em toneladas, para os anos de 1991 a 2000, utilizou-se um conversor de unidade de medida, para transformar as diversas unidades de medidas de grandeza (i.e, mil cachos, mil frutos) para tonelada.

É importante, ainda, ressaltar que foram utilizadas na análise variáveis relativas, pois variáveis absolutas podem levar ao engano na interpretação dos resultados, pois costumam estar correlacionadas ao tamanho da população ou da área das regiões em estudo. Para tanto, foram construídos indicadores parciais de produtividade agrícola média da terra, formados pela razão entre a produção agrícola total (em tonelada) e a área plantada (em hectares) em nível de microrregião para todos os anos entre 1991 e 2003. 


\section{Análise exploratóra de dados espaciais}

\subsection{Idéias Gerais}

A análise exploratória de dados espaciais (AEDE) trata diretamente de efeitos decorrentes da dependência espacial e da heterogeneidade espacial. Em outras palavras, o objetivo desse método é descrever a distribuição espacial, os padrões de associação espacial (clusters espaciais), verificar a existência de diferentes regimes espaciais ou outras formas de instabilidade espacial (não-estacionariedade) e identificar observações atípicas (i.e. outliers).

Para implementar a AEDE, assim como para aplicar as técnicas de econometria espacial, como será visto mais adiante, é preciso, primeiramente, definir uma matriz de pesos espaciais (W). Essa matriz é a forma de se expressar um determinado arranjo espacial das interações resultantes do fenômeno a ser estudado. Por exemplo, é razoável supor que, no estudo de vários fenômenos, regiões vizinhas tenham uma interação mais forte entre si do que regiões que não são contíguas. De modo similar, regiões distantes entre si teriam uma interação menor. Nesse caso, em que a distância entre as regiões importa na definição da força da interação, seria possível construir uma matriz W baseada na distância inversa entre as regiões a fim de capturar tal arranjo espacial da interação. A escolha da matriz de pesos espaciais é muito importante em uma AEDE, pois os resultados da análise são sensíveis a tal seleção. ${ }^{4}$

A matriz de peso espacial $W$, utilizada neste trabalho, está baseada na idéia dos $k$ vizinhos mais próximos, e calculada utilizando a métrica baseada no grande círculo entre os centros das regiões. ${ }^{5}$ Formalmente, tal matriz de pesos espaciais é expressa como:

\footnotetext{
${ }^{4}$ Qualquer matriz de pesos espaciais precisa atender às condições de regularidade impostas pela necessidade de invocar as propriedades assintóticas dos estimadores e dos testes. Segundo Anselin (1988), "isso significa que os pesos precisam ser não-negativos e finitos e que correspondam a uma determinada métrica".

${ }^{5}$ De acordo com Le Gallo e Ertur (2003), a escolha de um número fixo de vizinhos mais próximos ao invés do uso de uma matriz simples de contigüidade é preferível, pois é possível evitar alguns problemas metodológicos que podem ocorrer quando há variações no número de vizinhos.
} 


$$
\left\{\begin{array}{l}
w_{i j}(k)=0 \text { se } i=j \\
w_{i j}(k)=1 \text { se } d_{i j} \leq D_{i}(k) \text { e } w_{i j}^{*}(k)=w_{i j}(k) / \sum_{j} w_{i j}(k) \text { para } k=1,2, \ldots, n \\
w_{i j}(k)=0 \text { se } d_{i j}>D_{i}(k)
\end{array}\right.
$$

na qual $d_{i j}$ é a distância, medida pelo grande círculo, entre os centros das regiões $i$ e $j . D_{i}(k)$ denota um valor crítico que define o valor de corte para cada região, ou seja, a distância máxima para considerar regiões vizinhas à região $i$. Ou seja, distâncias acima deste ponto não serão tomadas como vizinhas da região em questão. ${ }^{6}$

Outro mecanismo muito útil para implementar a AEDE e a econometria espacial é o operador de defasagem espacial. ${ }^{7}$ A solução apresentada na literatura para se construir esse operador consiste na utilização de pesos espaciais para se obter uma medida de variável defasada para uma dada região. O operador de defasagem espacial para uma variável qualquer $y$, formalmente $W y$, pode ser interpretado como sendo a média do valor dessa variável nas regiões vizinhas. ${ }^{8}$

\subsection{Autocorrelação Espacial Global}

A autocorrelação espacial pode ser calculada por meio da estatística I de Moran. Essa estatística fornece a indicação formal do grau de associação linear entre os vetores de valores observados de uma variável de interesse no tempo $t\left(z_{t}\right)$ e a média ponderada dos valores da vizinhança, ou as defasagens espaciais $\left(W z_{t}\right)$. Valores de $I$ maiores (ou menores) do que o valor esperado $E(I)=-1 /(n-1)$ significa que há

\footnotetext{
${ }^{6}$ Neste estudo, foi utilizado valor de $k=15$ (ie., quinze vizinhos mais próximos), todavia, a fim de verificar a robustez dos resultados foi testado $\mathrm{k}=10$ e $\mathrm{k}=20$. Vale destacar que os resultados $\mathrm{k}=10 \mathrm{e} \mathrm{k}=20$ vizinhos mais próximos estão disponíveis para consulta.

7 O operador defasagem espacial é oriundo da idéia de defasagem temporal da econometria de séries de tempo. Contudo, o conceito de operador de defasagem espacial não é tão simples e direto como o operador de defasagem em séries de tempo devido à natureza multidirecional do processo de interação no espaço.

${ }^{8}$ Note-se que a matriz de pesos espaciais $W$ tem dimensão $n$ por $n$, sendo $n$ o número de unidades espaciais (por ex., microrregiões), ao passo que $y$ é um vetor-coluna com os valores da variável de interesse (por exemplo, produtividade agrícola média).
} 
autocorrelação positiva (ou negativa). Seguindo Cliff e Ord (1981), em termos formais, a estatística I de Moran pode ser expressa como:

$$
I_{t}=\left(\frac{n}{S_{o}}\right)\left(\frac{z_{t}^{\prime} W z_{t}}{z_{t}^{\prime} z_{t}}\right) t=1, \ldots n
$$

em que $z_{t}$ é o vetor de $n$ observações para o ano $t$ na forma de desvio em relação à média. Mais uma vez, $W$ é a matriz de pesos espaciais, sendo que os elementos $w_{i i}$ na diagonal são iguais a zero, enquanto que os elementos $w_{i j}$ indicam a forma como a região $i$ está espacialmente conectada com a região $j$. O termo $S_{o}$ é um escalar igual à soma de todos os elementos de $W$.

Quando a matriz de pesos espaciais é normalizada na linha, isto é, quando os elementos de cada linha somam um, a expressão (3) assume a seguinte forma:

$$
I_{t}=\left(\frac{z_{t}^{\prime} W z_{t}}{z_{t}^{\prime} z_{t}}\right) t=1, . . n
$$

A autocorrelação espacial positiva indica que microrregiões que apresentam elevada produtividade média agrícola são vizinhas de outras microrregiões que também apresentam elevada produtividade média agrícola. Ou, alternativamente, que microrregiões com baixa produtividade média agrícola são circundadas por outras microrregiões também ostentando baixa produtividade média.

A Tabela 2 mostra os resultados do Índice de Moran, o desvio padrão e o $z$-value para a produtividade média agrícola para os anos selecionados de 1991, 1997 e 2003. Como podem ser observados, são positivos e crescentes todos os valores de uma variável de interesse qualquer $z$ quanto do I de Moran, o que identifica uma autocorrelação espacial global positiva em todos os períodos.

Cabe ressaltar que a estatística I de Moran, utilizando o conceito de $k$ vizinhos mais próximos para os valores de vizinhos utilizados neste trabalho (isto é, quinze e vinte), leva ao mesmo resultado em relação ao sinal e à significância da autocorrelação espacial, ou seja, os resultados são robustos em relação à escolha da matriz de pesos. 
Tabela 2. I de Moran, desvio padrão e z-value para a produtividade média agrícola brasileira para os anos de 1991, 1997 e 2003

\begin{tabular}{ccccc}
\hline Ano & $I$ de Moran & Média & Desvio Padrão & $z$ - value \\
\hline 1991 & 0,4539 & $-0,002$ & 0,008119 & 63,468407 \\
1997 & 0,5623 & $-0,002$ & 0,008119 & 63,468407 \\
2003 & 0,5651 & $-0,002$ & 0,008119 & 63,468407 \\
\hline
\end{tabular}

Fonte: elaboração dos autores.

\subsection{Diagramas de Dispersão de Moran}

Segundo Anselin (1996), o diagrama de dispersão de Moran é uma das formas de interpretar a estatística $I$ de Moran. Trata-se de uma representação do coeficiente de regressão e permite visualizar a correlação linear entre $z$ e $W z$ através do gráfico de duas variáveis. A regressão é especificada de forma que o coeficiente $I$ de Moran é a inclinação da curva de regressão de $W z$ contra $z$, sendo que essa inclinação indica o grau de ajustamento.

O diagrama de dispersão de Moran é dividido em quatro quadrantes (AA, BB, AB e BA). Esses quadrantes correspondem a quatro padrões de associação local espacial entre as regiões e seus vizinhos.

As regiões localizadas nos quadrantes AA e BB apresentam autocorrelação espacial positiva, ou seja, essas regiões formam clusters de valores similares. Por outro lado, os quadrantes BA e AB apresentam autocorrelação espacial negativa, ou seja, estas regiões formam clusters com valores diferentes.

O diagrama de dispersão de Moran tem uma limitação devido à ausência da avaliação da incerteza estatística dos clusters AA, BA, BB e AB.

O diagrama de dispersão de Moran ${ }^{9}$ (Figura 1) apresenta no eixo horizontal a produtividade média para 1991 (PME91),respectivamente,e, na vertical, a defasagem espacial da variável de interesse para o mesmo período (W_PME91).

\footnotetext{
${ }^{9}$ Todos os diagramas de dispersão de Moran estão disponíveis para consulta.
} 
Figura 1. Diagrama de Dispersão de Moran da Produtividade Média Agrícola Brasileira para o ano de 1991

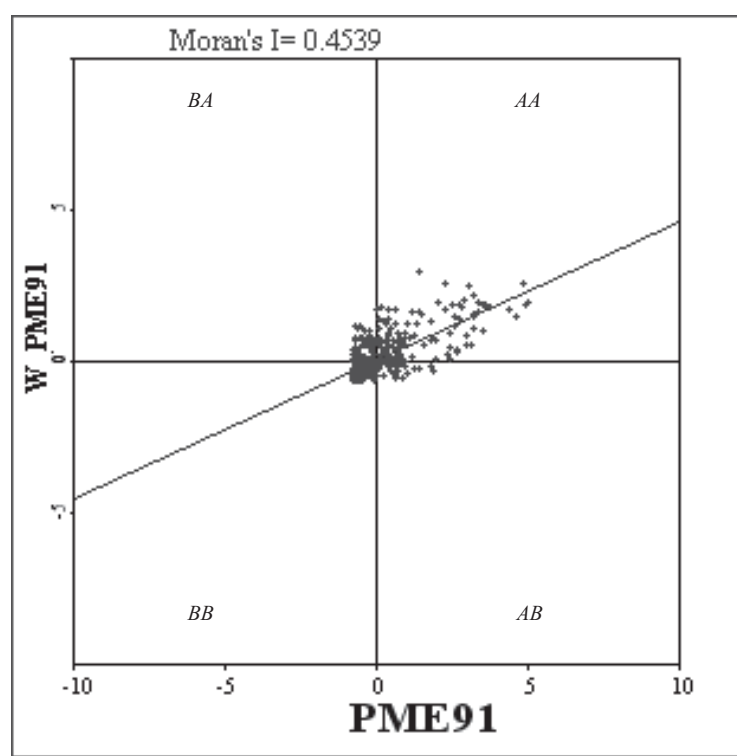

Fonte: resultado da pesquisa.

Os resultados de autocorrelação espacial (I de Moran), mostrados na seção 3.2, podem ser corroborados pelo fato de que a maioria das regiões está localizada nos quadrantes AA e BB para todos os anos avaliados no presente trabalho. Para o ano de 1991, por exemplo, observase que $76 \%$ das microrregiões são caracterizadas por valores similares ( $23 \%$ no quadrante AA e $52 \%$ no quadrante BB). Desse modo, a autocorrelação espacial positiva manifesta-se mais na forma de clusters com microrregiões de baixa produtividade do que clusters de alta produtividade agrícola.

\section{Análise de convergência espacial}

A definição de um modelo para captar, por exemplo, questões inerentes a efeitos de transbordamento entre as regiões, devem considerar explicitamente componentes espaciais em sua forma funcional. Portanto, os modelos tradicionais de regressão linear, que não levam em conta 
os efeitos espaciais (autocorrelação e heterogeneidade espaciais), não devem ser estimados por intermédio do método dos mínimos quadrados ordinários, pois as estimativas serão inconsistentes e/ou ineficientes. ${ }^{10}$

Assim sendo, ao estimar por mínimos quadrados ordinários (MQO), o modelo de $\beta$ convergência, busca-se apenas identificar qual é a melhor maneira de se estimar a Equação 5, dada como:

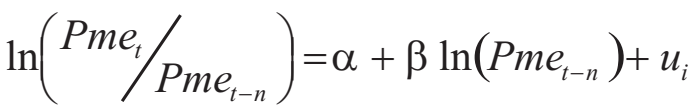

na qual $\ln \left(\mathrm{Pme}_{t} / P m e_{t-n}\right)$ é o logaritmo natural da razão entre a produtividade média entre dois anos em análise (e.g., produtividade média em 2003 e produtividade média em 1991); $\beta \ln \left(P m e_{t-n}\right)$ é o logaritmo natural da produtividade média no período inicial e $u_{i}$ é o termo de erro.

A fim de identificar a melhor especificação do modelo de $\beta$ convergência, levando em conta autocorrelação espacial, segue-se a proposta feita por Florax, Folmer e Rey (2003), ou seja, como primeiro estágio, os autores recomendam que seja seguido o roteiro:

a) Estimar o modelo clássico de análise de regressão linear por MQO;

b) Testar a hipótese de ausência de autocorrelação espacial devido a uma defasagem ou a um erro por meio do valor do multiplicador de Lagrange para defasagem espacial $\left(\mathrm{ML}_{\rho}\right)$ e Multiplicador de Lagrange para o erro espacial $\left(\mathrm{ML}_{\lambda}\right)$;

c) Caso ambos os testes não sejam significativos, a utilização do modelo clássico é mais apropriada. Caso contrário, é necessário seguir o próximo passo;

d) Caso ambos sejam significativos, estima-se o modelo apontado como o mais significante de acordo com as versões robustas desses testes, ou seja, o multiplicador de Lagrange robusto para a defasagem espacial $\left(\mathrm{MLR}_{\rho}\right)$ e o multiplicador de Lagrange robusto para o erro espacial $\left(M_{L} R_{\lambda}\right)$. Caso $\operatorname{MLR}_{\rho}>M_{\lambda} R_{\lambda}$ identificase o modelo com defasagem espacial como o mais apropriado. Caso contrário, $M_{L} R_{\rho}<M_{\lambda}$, adota-se o modelo de erro espacial como o mais apropriado.

${ }^{10}$ A esse respeito, ver Anselin (1988) e Anselin e Bera (1998). 


\subsection{Modelos}

\subsubsection{Modelo de Erro Espacial}

A primeira modificação pode ser o caso em que o termo de erro $u_{i}$ na Equação 5 segue um processo espacial autoregressivo, como mostrado na Equação 6:

$$
u_{i}=\lambda W u_{i}+\varepsilon_{i}
$$

na qual $\lambda$ representa o coeficiente escalar do erro espacial, enquanto o termo de erro ${ }^{\varepsilon_{i}}$ é normalmente distribuído com média zero e variância constante. Substituindo a Equação (6) na Equação (5) obtém-se a forma funcional do modelo de regressão do erro espacial:

$$
\ln \left(\mathrm{Pme}_{t} / \mathrm{Pme}_{t-n}\right)=\alpha+\beta \ln \left(\mathrm{Pme}_{t-n}\right)+(\mathrm{I}-\lambda W) \varepsilon_{\underline{i}}
$$

Note-se que a matriz $W$ é a mesma matriz de contigüidade utilizada no cálculo da estatística I de Moran. Quando $\lambda$ assumir o valor nulo, não existe autocorrelação espacial do erro. De acordo com Rey e Montouri (1999), quando $\lambda \neq 0$, um choque ocorrido em uma unidade geográfica se espalha não só para os seus vizinhos imediatos, mas por todas as outras unidades. Esse tipo de dependência espacial poderia ser resultante de efeitos nãomodelados que não fossem aleatoriamente distribuídos através do espaço.

\subsubsection{Modelo de Defasagem Espacial}

Neste modelo, a autocorrelação espacial é considerada como sendo gerada pela interação atual entre as regiões. Nesse caso, é introduzida uma defasagem espacial como variável independente na equação de convergência $\beta$. O modelo é especificado da seguinte forma:

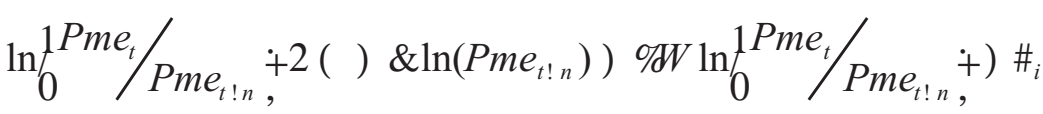

Note-se que $\rho$ é o coeficiente de defasagem espacial (um escalar). $\mathrm{O}$ elemento novo nesta forma funcional pode ser entendido como uma média dos valores da taxa de crescimento das regiões vizinhas. Espera-se que $\rho>0$, sugerindo a existência de autocorrelação espacial positiva. 


\subsubsection{Modelo Regressivo Cruzado Espacial}

Trata-se de um modelo que inclui efeitos de transbordamento espacial. No contexto da convergência, o efeito de transbordamento é representado pela defasagem espacial da produtividade média agrícola na terra do período inicial (1991). Assim, formalmente, o modelo assume a seguinte expressão:

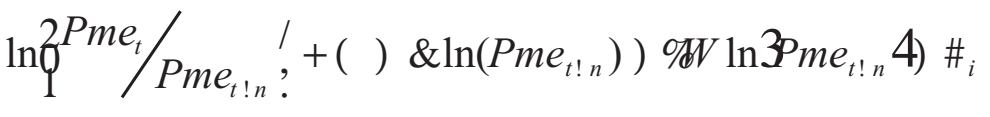

na qual $\tau$ é o coeficiente de transbordamento espacial, $W \ln \rrbracket m e_{t \square n}[$ denota a defasagem espacial da produtividade no período inicial (e.g. produtividade média em 1995) e $\varepsilon_{i}$ representa o termo de erro bem comportado.

Nesse modelo, conforme apontado por Rey e Montouri (1999, p. 151), a dependência espacial remanescente toma a forma da média da produtividade média agrícola do começo do período nas microrregiões vizinhas, que seria o termo de transbordamento cruzado. ${ }^{11}$ Note que $\tau$ é, nesse caso específico, um escalar. ${ }^{12}$

\subsection{Resultados e Discussão ${ }^{13}$}

A estimação da Equação (5), apresentada na seção 4, permite obter a estimativa do parâmetro da convergência $\beta$, que estuda a hipótese de que microrregiões com produtividade média agrícola baixa tendem a crescer mais rapidamente que microrregiões com produtividade média agrícola maior. Convém relembrar que a Equação 5 faz referência ao modelo de convergência absoluta.

Ao seguir os passos do procedimento de especificação, citado na seção 4, estima-se a Equação 5 por MQO. Os resultados estão reportados na Tabela 3.

\footnotetext{
${ }^{11}$ Considerando $\ln \left(P m e 91_{i}\right)$ como uma variável exógena, assim o é a variável defasada espacialmente $W \ln \left(P m e 91_{i}\right)$. Portanto, é possível estimar esse modelo por intermédio dos mínimos quadrados ordinários (Rey e Montouri, 1999, p. 151).

${ }^{12}$ Se houvesse efeitos de transbordamentos de outras variáveis explicativas, $\tau$ seria um vetor e não um escalar.

${ }^{13}$ É importante observar que o presente trabalho estima apenas a convergência para produtividade agrícola, e não quais são os fatores responsáveis pelo aumento dessa produtividade.
} 
Tabela 3. Resultados Econométricos para cada sub-período: 1991- 94; 1995-99; 2000-03; e para o período: 1991 a 2003

\begin{tabular}{ccccc}
\hline & $1991 / 1994$ & $1995 / 1999$ & $2000 / 2003$ & $1991 / 2003$ \\
\hline$\alpha$ & 0,1725 & $-0,0980$ & $-0,0125$ & 0,3150 \\
$\beta$ & $(0.0000)$ & $(0.0009)$ & $(0.6487)$ & $(0.0000)$ \\
AIC & $-0,0796$ & 0,0607 & 0,0239 & $-0,0674$ \\
SC & $(0.0000)$ & $(0.0000)$ & $(0.0803)$ & $(0.0024)$ \\
I de Moran & 623,8100 & 512,8350 & 407,9800 & 950,3100 \\
ML - Erro & 632,4590 & 521,4830 & 416,6300 & 958,9600 \\
& 0,1825 & 0,1846 & 0,1193 & 0,3379 \\
ML - defasagem & $(0.0000)$ & $(0.0000)$ & $(0.0000)$ & $(0.0000)$ \\
& 139,4400 & 142,6800 & 59,6220 & 477,8720 \\
MLR - Erro & $(0.0000)$ & $(0.0000)$ & $(0.0000)$ & $(0.0000)$ \\
& 116,6120 & 159,4390 & 62,1440 & 441,8720 \\
Teste Jarque - Bera & $(0.0000)$ & $(0.0000)$ & $(0.0000)$ & $(0.0000)$ \\
& 29,1930 & 3,6830 & 1,7871 & 47,5900 \\
& $(0.0000)$ & $(0.0549)$ & $(0.1812)$ & $(0.0000)$ \\
MLR - Defasagem & 6,3650 & 20,4380 & 4,3090 & 11,6286 \\
& $(0.0110)$ & $(0.0000)$ & $(0.0379)$ & $(0.0006)$ \\
& 2,3152 & 23,8400 & 9,4900 & 11,4020 \\
& $(0.1281)$ & $(0.0000)$ & $(0.0029)$ & $(0.0000)$ \\
& $(0.0000)$ & $(0.0000)$ & $(0.0000)$ & $(0.0000)$ \\
\hline
\end{tabular}

Fonte: Elaboração dos autores.

Nota: os resultados entre parênteses indicam o valor da probabilidade.

O conjunto de testes para averiguar a presença de autocorrelação espacial é útil tanto para auxiliar a identificação do modelo econométrico espacial quanto para a tarefa de validação ou diagnóstico desse modelo. Para tal, fez-se uso dos testes de multiplicador de Lagrange e multiplicador de Lagrange robusto.

É importante salientar dois problemas que podem surgir nos modelos estimados. Primeiro, a existência (da) heterocedasticidade, conforme se verifica aplicando o teste Breusch-Pagan. Segundo, os erros não 
seguem uma distribuição normal, de acordo com o teste Jarque-Bera. Ambos os problemas sugerem cautela com as inferências.

Para os sub-períodos 1991 a 1994 e 1991 a 2003, o melhor modelo é o do erro espacial. Tal resultado pode ser corroborado pela observação dos resultados quando se aplicam critérios de informação Akaike Information Criterion (AIC), Schwarz Criterion (SC) e do valor da função de verossimilhança (LIK), apresentados na Tabela 3.

Contudo, para o período de 1991-03 foram encontrados ambos os problemas citados acima (não-normalidade dos erros e heterocedasticidade) e para o sub-período 1991-94, apenas os erros não são normais.

Para corrigir a ausência de homocedasticidade, a especificação do modelo de erro espacial foi modificada para acomodar a heterocedasticidade na forma de grupos (groupwise heteroskedasticity).

Quanto ao problema da ausência de normalidade nos erros, adotouse o método de momentos generalizados (MG), proposto por Kelejian e Prucha (1999), para estimar o modelo de erro espacial, pois prescinde da hipótese da normalidade dos erros.

Já para os sub-períodos 1995-1999 e 2000-2003, o melhor modelo é o de defasagem espacial como podem ser confirmados por intermédio dos critérios de informação Akaike Information Criterion (AIC), Schwarz Criterion (SC) e o valor da função de verossimilhança (LIK). Tais resultados podem ser observados na Tabela 3.

Existem, no entanto, dois problemas recorrentes nos modelos estimados para os dois sub-períodos. Em primeiro lugar, existe heterocedasticidade, e em segundo, os erros não são normais.

Para corrigir a ausência de homocedasticidade, a especificação do modelo de defasagem espacial foi modificada para acomodar a heterocedasticidade na forma de grupos (groupwise heteroskedasticity), como feito para os sub-períodos 1991 a1994 e 1991 a 2003.

Quanto ao problema da ausência de normalidade nos erros, estimouse o modelo por Variáveis Instrumentais (VI), uma vez que essa estimação prescinde da normalidade dos erros.

Após seguir todos os critérios brevemente explicitados, foi possível estimar os modelos de convergência $\beta$, tal como pode ser observado na Tabela 4. 
Tabela 4. Resultados econométricos do modelo de erro espacial e do modelo de defasagem espacial para cada sub-período: 1991- 94; 1991-2003; 1995-1999; e 2000-2003

\begin{tabular}{|c|c|c|c|c|}
\hline \multirow[t]{2}{*}{ Coeficientes } & \multicolumn{2}{|c|}{ Modelo de Erro Espacial } & \multicolumn{2}{|c|}{ Modelo de Defasagem Espacial } \\
\hline & $1991 / 1994$ & $1991 / 2003$ & $1995 / 1999$ & $2000 / 2003$ \\
\hline$\alpha$ & $\begin{array}{c}0,1945 \\
(0.0000)\end{array}$ & $\begin{array}{c}0,3108 \\
(0.0000)\end{array}$ & $\begin{array}{l}-0,0663 \\
(0.0264)\end{array}$ & $\begin{array}{l}-0,0157 \\
(0.5575)\end{array}$ \\
\hline$\beta$ & $\begin{array}{l}-0,0894 \\
(0.7800)\end{array}$ & $\begin{array}{l}-0,0793 \\
(0.0000)\end{array}$ & $\begin{array}{c}0,0286 \\
(0.0885)\end{array}$ & $\begin{array}{c}0,0257 \\
(0.0510)\end{array}$ \\
\hline$\rho$ & & & $\begin{array}{c}0,0391 \\
(0.0000)\end{array}$ & $\begin{array}{c}0,0104 \\
(0.0000)\end{array}$ \\
\hline$\lambda$ & $\begin{array}{c}0,0257 \\
(0.0000)\end{array}$ & $\begin{array}{c}0,0218 \\
(0.0000)\end{array}$ & & \\
\hline Teste Breusch-Pagan & $\begin{array}{c}2,3152 \\
(0.1281)\end{array}$ & $\begin{array}{c}1,8972 \\
(0.1683)\end{array}$ & $\begin{array}{c}0,3557 \\
(0.5508)\end{array}$ & $\begin{array}{c}0,0916 \\
(0.7621)\end{array}$ \\
\hline
\end{tabular}

Fonte: elaboração dos autores.

Nota: os resultados entre parênteses indicam o valor da probabilidade.

Assim, ao analisar a estimativa do parâmetro $\beta$ para ambos os modelos, torna-se possível observar a existência ou não de convergência em relação à produtividade média agrícola brasileira. ${ }^{14}$

Primeiramente, ao analisar os sub-períodos 1995-1999 e 2000-2003 observa-se que os parâmetros estimados pelo modelo não são significativos estatisticamente. Portanto, pode-se afirmar que em ambos os sub-períodos, a taxa de convergência é nula.

Em segundo lugar, analisa-se a taxa de convergência para os períodos de 1991 a 1994 e 1991 a 2003, já que, como podem ser observados na tabela 4 , os resultados de convergência para tais períodos são

${ }^{14}$ A taxa de convergência $\theta$ foi computada segundo a fórmula: $\theta=\ln (\beta+1) /(-\mathrm{k})$. A respeito disso, consulte Rey e Montouri (1999, p. 152). 
significativos estatisticamente e por isso, cabe fazer a análise sobre a velocidade de convergência.

No período entre os anos de 1991 a 2003, a taxa de convergência é de 0,00635, portanto, ocorre a um passo lento. Todavia, é importante observar que o sub-período entre os anos de 1991 a 1994 foi o que mais contribuiu para a convergência da produtividade agrícola no Brasil, com uma taxa de convergência de 0,02193 . Ou seja, o período pós-abertura econômica foi o que mais contribuiu para diminuir as desigualdades de produtividade agrícola no Brasil.

\section{Considerações finais}

O artigo proporciona evidências sobre a evolução da produtividade agrícola brasileira, aplicando-se uma análise do grau de convergência $\beta$. No período entre 1991 e 2003, o estudo apresenta evidências em favor da existência de convergência absoluta da produtividade agrícola brasileira através das microrregiões. Isso sugere que a produtividade agrícola brasileira está convergindo para a média, porém a uma taxa muito lenta.

Portanto, cabe indagar quais são as implicações desse resultado para a economia brasileira e, principalmente, em qual proporção tal constatação pode influenciar o setor agrícola no longo prazo.

Ao observar uma convergência lenta na produtividade média agrícola, evidencia-se que a disparidade relacionada à produtividade média agrícola no Brasil, observada no item 2, está diminuindo de forma extremamente lenta durante os anos, colaborando para o aumento das disparidades regionais.

Portanto, a mensuração da convergência $\beta$ no presente artigo pode ser tomada como inovadora, com relação ao Brasil, sob dois aspectos: a) a incorporação dos efeitos espaciais explícitos no modelo; ou seja, mensura a convergência do setor agrícola, levando em conta a interação das regiões com o seu entorno, e b) em contraste com a grande maioria dos trabalhos, a utilização da produtividade como variável de convergência em detrimento da variável renda per capita.

Os resultados sugerem que pode ser interessante a realização de uma análise de convergência $\beta$ condicional, incorporando variáveis explicativas como estoques de capital físico, de capital humano e de fatores edafo-climáticos. 


\section{Referências bibliográficas}

ANSELIN, L. Interactive techniques and exploratory spatial data analisys. Longley P. A, Goodchild M.F, Maguire D.J e Wind D. W (eds). Geographical information system: principles, techniques, management and applications. Wiley, New York. p 253-365. 1998

ANSELIN, L. Spatial econometrics: methods and models. Kluwer Academic, Boston, 1988.

ANSELIN, L. e BERA, A. Spatial dependence in linear regression models with an introduction to spatial econometrics. In: Ullah A. and Giles D. E. (eds.) Handbook of Applied Economic Statistics. Marcel Dekker, New York, p. 237-289, 1998.

ANSELIN, L The Moran scatterplot as an ESDA tool to assess local instability in spatial association. Fisher, M, Scholten, H.J e Unwin, D W (eds). Spatial analytical perspectives in GIS. Taylor\&Francis. London. p 111-125. 1996.

ANSELIN, L. Local indicators of spatial association - LISA. Geographical Analysis, v. 27, n. 2, abril. p. 93-115, 1995.

BARRO, R. e SALA-I-MARTIN, X. Economic growth. MacGraw Hill. New York, 1995.

BAUMOL, W. J. Productivity growth, convergency, and welfare: What the long-run show. American Economic Review, v. 76, n. 5, p. 107285, 1986.

CLIFF, A. D. e ORD, J.K. Spatial processes: models and applications. Pion, London. 1981.

CONSIDERA, C. M., SOUSA, E. L. L. e BRACALE, G. Âncora verde: o papel da agricultura no ajuste econômico. SEAE, Brasília, Distrito Federal, 2002.

DALL'ERBA, S. Productivity convergence and spatial dependence among Spanish regions. Texto para discussão REAL/UIUC, Illinois, 2003.

DEPARTAMENTO INTERSINDICAL DE ESTATÍTICAS E ESTUDOS SÓCIO-ECONÔMICOS - DIEESE. Dez anos do Real. São Paulo, 2004. 
FIGUEIREDO, A. M. R. Resposta da produção agrícola aos preços no Centro-Oeste brasileiro: uma análise de econometria espacial para o período 1975-1995/1996. Tese de Doutorado, Universidade Federal de Viçosa, 2002. (mimeo).

FLORAX, R. J. G. M., FOLMER, H., REY, S. J. Specification searches in spatial econometrics: The relevance of Hendry's methodology. Regional Science and Urban Economics, vol. 33, n. 5, p. 557-79, 2003.

JOHNSTON, B.F.; MELLOR, J.W. The role of agriculture in economic development. American Economic Review, vol. 51, n.4, p. 566-93, 1961.

KELEJIAN, H. H. e PRUCHA, I. R. (1999). A generalized moments estimator for the autoregressive parameter in a spatial model. International Economic Review, vol. 40, n. 2.

LE GALLO, J and ERTHUR, C. Exploratory spatial data analysis of the distribution of regional per capita. GDP in Europe, 1980-1995. Papers in Regional Science, vol. 82, n. 2, p. 175-201. 2003.

LOPES, J. L. Avaliação do processo de convergência da produtividade da terra na agricultura brasileira no período de 1960 a 2001. Tese de Doutorado, ESALQ/USP, 2004. (mimeo).

MAGALHÃES, A. HEWINGS, G. J. D. AZZONI, C. R. Spatial dependence and regional convergence in Brazil. Texto para discussão, REAL/ UIUC, Illinois, 2000.

Ministério da Agricultura, Pecuária e Abastecimento (MAPA). Brasília, 2005. Disponível em: < http://www.agricultura.gov.br/ > Acesso em: 10 fev 2005.

QUAH, D. T. Regional convergence clusters across Europe. European Economic Review, vol. 40, p. 951-958, 1996.

REY, J. S., e MONTOURI, B. D. US Regional income convergence: a spatial sconometric perspective. Regional Studies, vol. 33, n. 2, p. 143156, 1999.

Sistema IBGE de Recuperação Automática (SIDRA). Brasília, 2005. Disponível em: < http://www.sidra.ibge.gov.br/bda/ > Acesso em: 5 fevereiro de 2005. 